\title{
Effect of Post-Exercise Yoga Poses on Eccentric Exercise-Induced Muscle Soreness, Pain, and Activities of Daily Living
}

\author{
Chakravarthy Marx Sadacharan \\ School of Biological Sciences (SBS), University of New England (UNE), Biddeford, Maine, 04005, United States
}

Received November 15, 2020; Revised December 25, 2020; Accepted January 20, 2021

\section{Cite This Paper in the following Citation Styles}

(a): [1] Chakravarthy Marx Sadacharan, "Effect of Post-Exercise Yoga Poses on Eccentric Exercise-Induced Muscle Soreness, Pain, and Activities of Daily Living," International Journal of Human Movement and Sports Sciences, Vol. 9, No. 1, pp. 19 - 26, 2021. DOI: 10.13189/saj.2021.090103.

(b): Chakravarthy Marx Sadacharan (2021). Effect of Post-Exercise Yoga Poses on Eccentric Exercise-Induced Muscle Soreness, Pain, and Activities of Daily Living. International Journal of Human Movement and Sports Sciences, 9(1), 19 26. DOI: 10.13189/saj.2021.090103.

Copyright $\bigcirc 2021$ by authors, all rights reserved. Authors agree that this article remains permanently open access under the terms of the Creative Commons Attribution License 4.0 International License

\begin{abstract}
The eccentric (ECC) exercises are an efficient way to exercise the muscles than concentric exercises. However, unaccustomed ECC may cause muscle damage, pain, delayed onset muscle soreness (DOMS) that may lead to temporary functional impairments. The aim is to measure the effects of performed yoga poses after various intensities of ECC exercise protocol with different ranges of motion (ROM) at the elbow and knee. Thirty-six participants aged between 18 and 30 years performed yoga after the ECC protocols (weeks $3,5,7,9$ ) and the ECC protocols without yoga poses (weeks 2, 4, 6, 8) on the elbow and knee muscles $(5 \times 10$ repetitions each session) using their $25 \% / 35 \%$ of maximal voluntary isometric contraction (MVIC). The subjective and objective assessments were carried out (activities of daily living (ADL), pain (visual analog scale (VAS), pain pressure threshold (PPT), and MVIC). The FROM ECC exercise with $35 \%$ of MVIC without yoga showed moderate pain (0-3.5) in ADL, VAS, and PPT for elbow extensors and showed a statistically significant difference $(\mathrm{p} \leq 0.05)$ compared to the PROM ECC exercise with $25 \%$ of MVIC without yoga. ECC exercise performed with 35\% of MVIC without yoga induced a higher degree of DOMS, pain, ADL limitations than 25\% of MVIC without yoga. Yoga after PROM ECC protocol showed less pain, DOMS in VAS, and PPT for elbow flexors, knee flexors, and knee extensors. The overall percentage of increased MVIC value at the end of the study was
\end{abstract}

between 30.53 and $43.12 \%$. The upper extremity (extensors) muscles are more prone to DOMS and pain when compared to the lower extremity muscles. Performing yoga stretching poses after the eccentric exercise protocol may affect reducing DOMS and muscle pain.

Keywords Exercise, Muscle, Pain, Soreness, Yoga

\section{Introduction}

Delayed onset muscle soreness (DOMS) or muscle pain occurs because of physical activity that strains the muscle tissue $[1,2]$. DOMS occurs when the muscle is performing a lengthening or an eccentric (ECC) contraction $[1,3]$. The severity of muscle damage is caused by the range of motion (ROM; muscle contraction length), intensity, and repetition [4, 5]. DOMS can be experienced by individuals in varying degrees depending upon their level of fitness, type, and intensity of the exercise performed. For example, a given set of exercise intensity may induce a less amount of DOMS in an athlete than a sedentary individual. In case of severe DOMS, individuals might experience pain, temporary discomfort, and difficulty in performing the activities of daily living (ADLs). Various interventions were used by the 
researchers to treat the DOMS [1, 5-7].

National Health Interview Survey data conducted by the Centers for Disease Control and Prevention (CDC) showed increased usage of complementary and alternative medicine (CAM) treatments. Yoga has gained popularity tremendously in the past years to treat many diseases. Yoga is a process of physical and mental training along with, simple meditation, breath control, and the adoption of specific body postures. It is widely practiced around the world for health and relaxation. Individuals performing yoga poses have tremendous health benefits like relief pain [8-12] and improve flexibility and balance [13], muscle strength $[14,15]$, skeletal muscle oxygen uptake [16], and lung function [17], and many more. At the gym, Yoga poses are recommended along with workout sessions. It can be either performed before or after the exercise protocol depending upon the intensity, type, and repetitions of the exercise protocol. In mild to severe intensity exercise protocol, certain types of yoga poses are recommended after the workout as relaxation and cool down. But still, it is not clear that the types of yoga poses can be performed after the ECC exercise protocol is recommended for sedentary individuals. There is a need for balance between the level of yoga poses that can be done after the various intensity ECC exercise protocol. In the published literature search, a study by Boyle et al. [18] showed that in women, yoga training, and a single bout of yoga following a short period of bench-stepping eccentric exercise greatly reduces the DOMS.

We hypothesized that the level of DOMS, pain, and functional impairment would be lower when certain yoga poses were performed after ECC exercise protocol, compared with ECC exercise performed without yoga poses. We choose specific yoga poses that involve elbow and knee muscle groups to see the effect of yoga poses performed after ECC exercise protocol.

The aim was to see the effects of performing yoga poses after various intensities of ECC exercise protocol with different range of motion (ROM) at elbow and knee.

\section{Materials and Methods}

\subsection{Participants}

Thirty-six untrained active healthy men and women were recruited after the screening procedure at the University of New England (UNE) students aged between 18 and 30 years.

\subsection{Screening}

Health screening (height, weight, body mass index, blood pressure, and heart rate) was carried out on the participants (Table 1). Inclusion criteria: (Participants must be): An untrained, active, healthy, man or woman (not involved in any kind of physical exercise protocols; involved in the day to day regular activities), and free of pain in the arms and legs. Exclusion criteria: Have had an injury in the arms and/or legs that caused a visit to a medical provider, high blood pressure (systolic $>130$ or diastolic $>90$ ), pregnant, diabetes (type 1 or type 2 ) and any nerve, muscle, or joint disorder. Participants could perform their normal activities of daily living (ADL) during the testing period and were asked to refrain from consuming any anti-inflammatory drugs and nutritional supplements two hours before the exercise protocol and alcohol 12 hours before the exercise protocol. The study has been approved by the authors' Institutional Review Board (20180508-004) and conducted in the Biology Research lab at Alfond Center for Health Sciences (ACHS) building and Campus Center Gym, UNE, Maine, USA.

\begin{tabular}{cc} 
Table 1. & Demographical characteristics of the study participants \\
\hline Participants & $\begin{array}{c}\text { Mean } \pm \text { SD } \\
\text { (standard deviation) }\end{array}$ \\
\hline Age (years) & $25.5 \pm 1.45$ \\
Height (cm) & $175.2 \pm 5.35$ \\
Bodyweight (Kg) & $66.21 \pm 6.27$ \\
Body mass index (BMI) & $23.45 \pm 2.70$ \\
Blood pressure (BP) & $120 / 84 \pm 3 / 2$ \\
Heart rate (HR) & $86 / \mathrm{min} \pm 11$
\end{tabular}

\subsection{Procedures}

This study was carried out for a period of nine weeks: Week 1 - pre-test screening, the familiarization session, and MVIC calculation. Every week, the participants were called for six days where only three days (days 1,3 , and 5 ) performed ECC exercises (5 sets of 10 repetitions for all four muscle groups) on elbow flexors, elbow extensors, knee flexors, and knee extensors and remaining days 2, 4, and 6, subjective ((ADLs, Visual analog scale (VAS) and objective (Pain pressure threshold (PPT), and muscle strength (MVIC)) assessments were carried out. Weeks 2, 3,4 , and 5 - elbow and knee ECC exercise protocol using their MVICs (25\% (week 2), yoga+25\% (week 3), 25\% (week 4) and yoga $+25 \%$ (week 5)) at a partial range of motion (PROM) $\left(60^{\circ}\right.$ of total ROM - an outer range of motion) and the full range of motion (FROM), subjective and objective assessments, and post ECC protocol MVIC calculation; Weeks 6, 7, 8, and 9 - elbow and knee ECC exercise protocol using their MVICs (35\% (week 6), yoga $+35 \%$ (week 7), 35\% (week 8) and 35\% (week 9)) at PROM and FROM, subjective and objective assessments, and post ECC protocol MVIC calculation. Weeks 2, 3, 6, and 7 ECC exercise protocol was performed with PROM whereas, weeks 4, 5, 8, and 9 ECC exercise protocol was performed with FROM. Yoga poses were also performed after each day of ECC exercise in weeks 3, 5, 7, and 9 (Figure 1). Familiarization: (week 1 - day 1 (visit 1)): The investigator reviewed the study and the informed consent form with participants. The study procedure including step 
by step to do maximal voluntary isometric contraction (MVIC) and eccentric (ECC) exercises in four of their muscle groups (elbow flexors, elbow extensors, knee flexors, and knee extensors), associated risks that may arise due to their participation, and the health benefits of the participants in this research project were explained.

\subsection{Yoga Poses}

The five yoga poses were selected and demonstrated using yoga mats to all the participants by the certified yoga instructors. The yoga poses were performed by the participants after each day of PROM and FROM ECC exercise protocol in weeks 3, 5, 7, and 9 (3 days per week). Yoga sessions were conducted as a group activity and repeated five times. All yoga sessions were started with a breathing exercise (6-8 times). A total duration of 20-30 mins was spent on each yoga session. The five yoga poses are: a. Child's pose- Participants sat on the heels, then slowly brought their forehead down to rest in front of their knees. Rested the arms down alongside the body and took eight deep breaths. b. Thread the needle pose: Participants sat on the heels and slid the right hand between the left hand and left knee. Slid the arm out to the left so that the right shoulder and side of the head rested comfortably on the floor. Then, inhaled and reached the left hand up towards the ceiling. At first, explored the posture with the arm, found the place where the participant felt the deepest stretch, then stayed there and reached out through the fingers. Breathed and held for 6-8 breaths. To release: exhaled the palm back to the floor and slowly inhaled back to sitting on the heels. The same was repeated on the other side. c. Eagle arms: Participants stood tall in mountain pose. Wrapped one leg around the other, brought their bent arms out in front and wrapped their arms together in the opposite way, and slightly bent knees. Perched on a tree-like an eagle. Breathed and held for 6-8 breaths. d. Cow face pose: Participants sat in a crossed-leg position, right leg over the left. Spread the legs as far apart as possible without bending their knees. The participant bent the left knee and placed the bottom of the left foot against the inner left thigh. Brought their left heel as close to the perineum as possible. Kept their left knee on the floor. After that, grasped the right foot with the left hand, and kept the foot on the floor, placed the heel of the right foot against the front-left portion of the left buttock. The right knee was directly on top of the left knee. Inhaled slowly through the nostrils and raised the right hand over the head and bent the right elbow. Reached behind the back with the left hand and clasped the fingers of both hands (forming an "s" shaped lock). Maintained the posture comfortably hold the inhaled breath. Later, exhaled slowly and then repeated the posture reversing the arms and legs. Breathed and held for 6-8 breaths. e. Hands overhead in wide-legged forward bend: From mountain pose, stepped their feet out wide and bent their upper body and clasped the hands together. Pretended that the participant's arms were the trunk of an elephant. Then, kept their hands overhead. Breathed and held for 6-8 breaths.

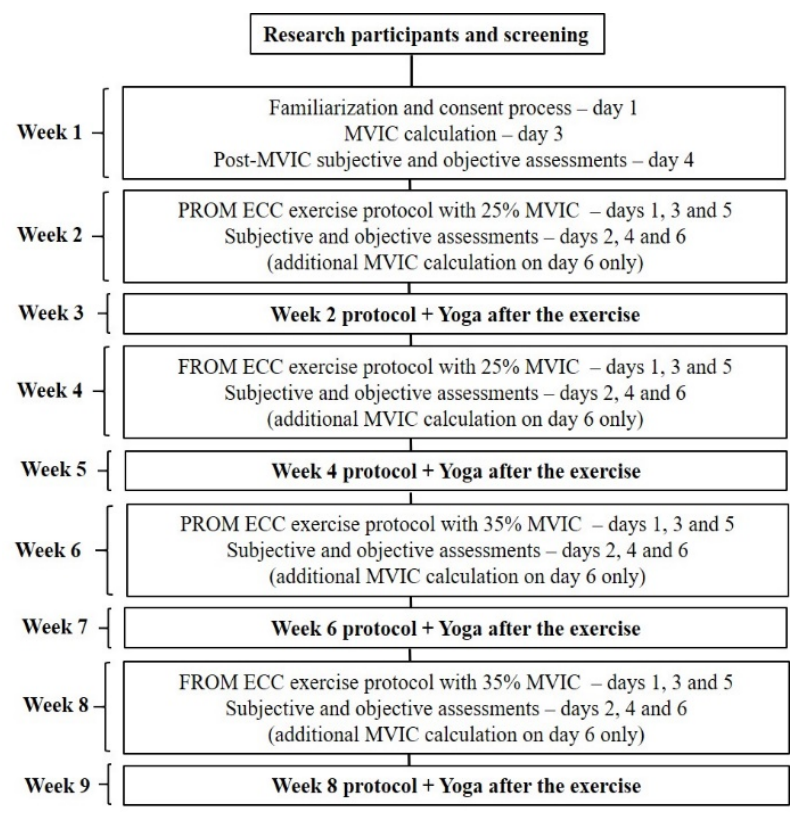

Figure 1. Simplified experimental design

The MVICs were performed on four selected muscles using a hand-held dynamometer (Lafayette Instrument, Manual Muscle Testing (MMT) Device, Pro-Health Care, USA) for 4 positions: 1) Elbow flexion at 90 degrees; 2) Elbow extension at 90 degrees; 3) Knee flexion at 90 degrees; and 4) Knee extension at 90 degrees. Depending on the visit, $25 \%$ or $35 \%$ of MVICs were calculated for the ECC exercise protocol.

Baseline delayed onset of muscle soreness (DOMS) assessment after MVIC testing: (week 1) After 24 hours of familiarization, the assessment of the ADL, DOMS, pain, and muscle strength were carried out.

\subsection{Subjective Assessments}

a. Health activities of daily living (ADL) difficulty scale - The following questions were asked. The pain scale ranged from 0 (no pain) to 10 (worst pain). For elbow: 1. Combing hair, 2. Pulling a heavy object, 3. Using arm to rise from a chair, 4. Carrying an object above the shoulder, 5. Putting on shirt/coat, 6. Washing opposite armpit and back for knee: 1 . Getting in and out of the car, 2. Walking on the flat ground, 3. Ascending stairs, 4. Descending stairs, 5. Getting in and out of bed, 6 . Bending to pick up from the floor.

a). Visual Analog Scale (VAS) - The level of muscle soreness was quantified using an 11 points VAS in which 0 indicated "no pain" and 10 represented "extreme pain". The participants were asked to mark the level of perceived soreness on the VAS when the 
elbow flexors, elbow extensors, knee flexors, and knee extensors are palpated in a circular motion by the investigator.

b). The delayed-onset of muscle soreness (DOMS) was assessed by asking the participants to perform the movements at the elbow and knee joints (flexion for extensors and extensors for flexors) and pain level was recorded based on the verbal response of the participants.

\subsection{Objective Assessment}

a). Pressure Pain Threshold (PPT) - PPT is the minimum amount of force that can be applied to induce pain, that was measured using an electronic algometer (Baseline 60-pound Dolorimeter/Algometer Pain Threshold Meter, Pro-Health Care, USA). The VAS and PPT measurements helped us to compare the research participants' pain responses in the induced DOMS.

\subsection{Eccentric (ECC) Exercise Protocol}

The calculated elbow and knee flexors or extensors, 25\% or $35 \%$ of MVIC was attached to the research participant's wrist and ankle using Ultimately Fit Adjustable Ankle Weights (Hayneedle Company, USA) and facilitated the PROM and FROM ECC exercise protocols. The dynamic adjustable ROM controlled elbow and knee joint splints (T Scope ${ }^{\circledR}$ Elbow Premier (07254) and T Scope ${ }^{\circledR}$ Premier Post-Op Knee Brace (08814), BREG, USA) were used to limit the partial ROM $\left(60^{\circ}\right.$ of total ROM - the outer range of motion) while performing ECC protocols with PROM. These splints have locks to control the ROM so unexpected or uncontrolled movements at the elbow and knee of the subjects were prevented. We did not use any splint or external devices while performing ECC protocols with FROM (weeks 6-9) (Figure 2 and 3).

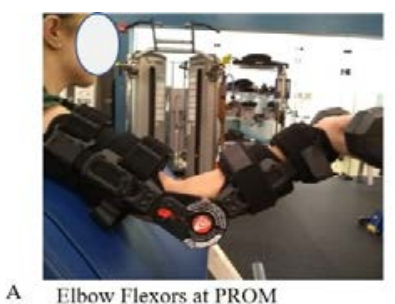

A Elbow Flexors at PROM

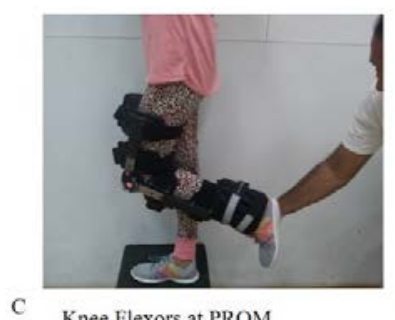

Knee Flexors at PROM

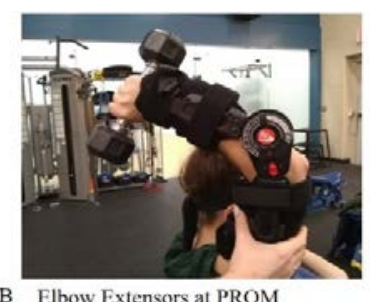

B Elbow Extensors at PROM

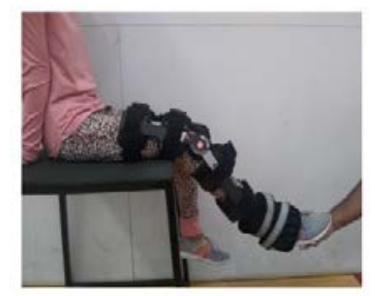

D Knee Extensors at PROM
Figure 2. PROM elbow and knee eccentric exercise positions; PROM partial range of motion
Exercise Protocol: Each week (week 2-9)- participants were introduced to ECC exercise on days 1, 3, and 5 (5 sets of 10 repetitions for all four muscle groups) at PROM/FROM with calculated $25 \% / 35 \%$ of MVIC. The yoga poses were also performed after each day of ECC exercise protocol in weeks $3,5,7$, and 9 (3 days per week).

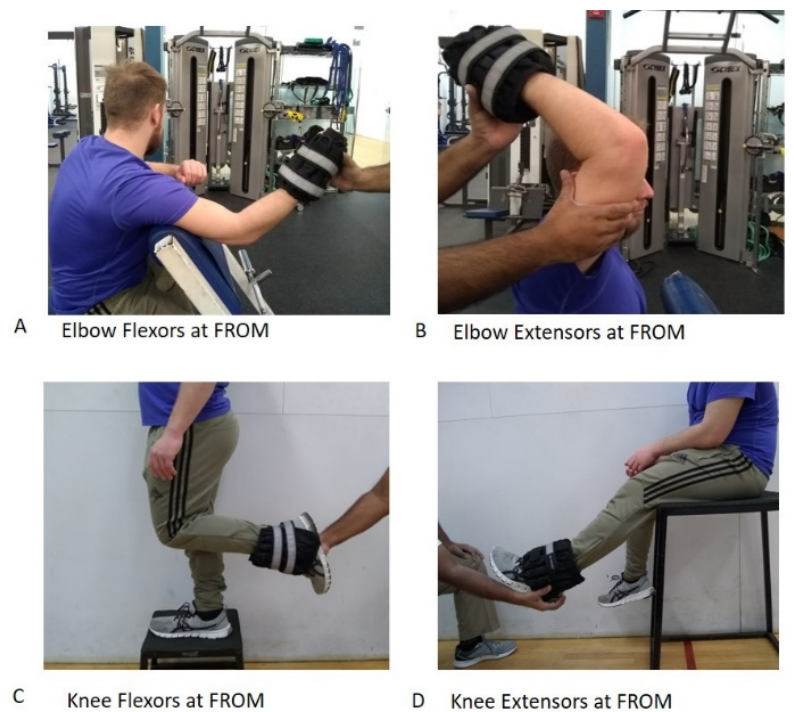

Figure 3. FROM elbow and knee eccentric exercise positions; FROM full range of motion

Post- ECC Exercise assessment: days 2, 4, and 6 - After every ECC exercise protocol, the subjective and the objective assessments were carried out for 24-48 hours to see the functional impairment involving elbow and knee joints. The muscle strength (MVIC) was calculated for all four muscle groups at the end of each week (Figure 1).

\subsection{Statistical Analysis}

Statistical analysis was carried out using GraphPad Prism software (version 8.2.0), San Diego, CA. The paired-sample t-test and repeated measures one-way ANOVA with Tukey's multiple comparison tests were carried out to compare the difference between pre-exercise, yoga after the PROM, and FROM ECC exercise protocols. Statistical significance was set at an alpha level at $\mathrm{p} \leq 0.05$.

\section{Results}

Yoga poses performed after the PROM ECC protocol (25\% of MVIC in week 3 ) showed less pain, soreness, and muscle strength when compared to FROM ECC protocol without yoga poses (35\% of MVIC in week 8). The ADL activities pain levels were less (pain level 0-0.5) and did not show any statistically significant difference between yoga after the PROM and yoga after the FROM ECC exercise weeks. 


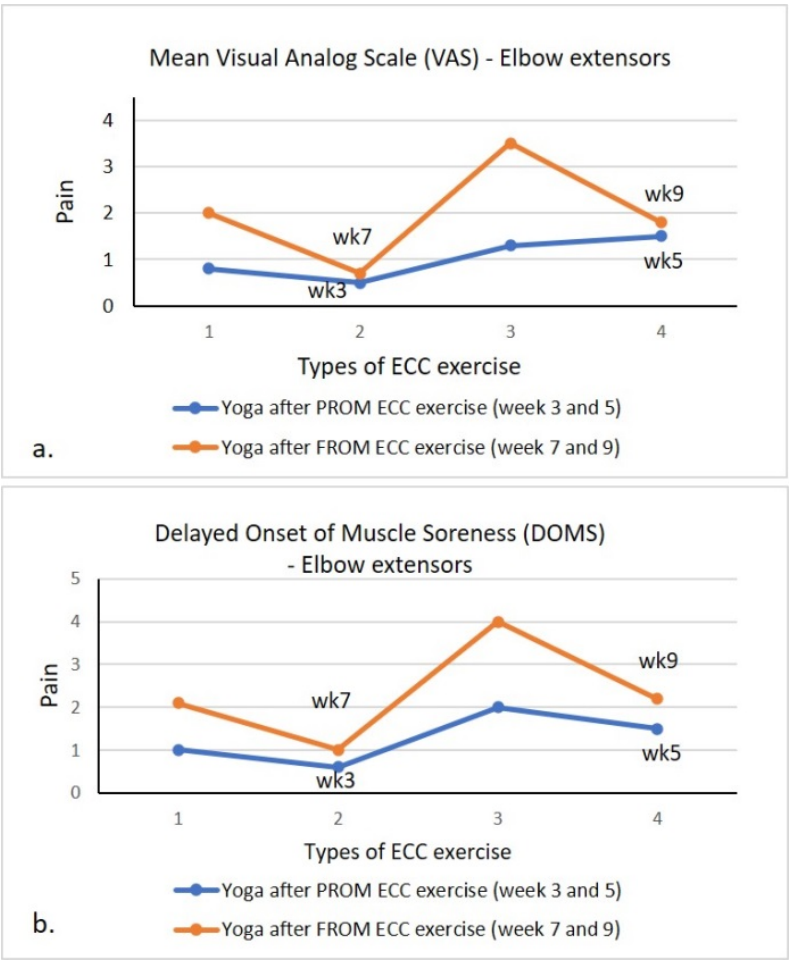

Figure 4. Visual analog scale (VAS) and delayed onset of muscle soreness (DOMS) comparison between yoga after the partial range of motion (PROM) and yoga after the full range of motion (FROM) eccentric exercise protocols

The pain level of VAS and DOMS assessments are shown in Figure 4. Yoga after the PROM ECC protocol (weeks 3 and 7) showed less pain on elbow extensors than yoga after the FROM ECC protocol (weeks 5 and 9) in both VAS and DOMS assessments. Yoga after the PROM ECC protocol (weeks 3 and 7) showed less pain on elbow extensors than PROM ECC protocol without yoga poses (weeks 2 and 6) in both VAS and DOMS assessments. FROM ECC protocol without yoga poses (week 4 and 8) showed a moderate pain (pain level 2-5) on elbow extensors than yoga after the FROM ECC protocol (week 5 and 9) in both VAS and DOMS assessments and did show a statistically significant difference $(\mathrm{P} \leq 0.05)$ between them. The elbow flexors, knee flexors, and knee extensors pain levels were less (pain level 0-0.5) and did not show any statistically significant difference between the ECC exercise weeks.

PPT was compared between yoga after the PROM and FROM exercise protocols (Figure 5). The moderate PPT (pain level 4.2) was measured at the mid-belly of triceps brachii in the FROM ECC protocol without yoga poses (35\% of MVIC) at week 8 and mild PPT (pain level 6.6) was measured in the yoga after the PROM ECC protocol (25\% of MVIC) at week 3. Mid-belly of the biceps brachii, mid-upper anterior thigh, and mid-upper posterior thigh PPT values showed minimal pain and did not show any statistically significant difference between them.

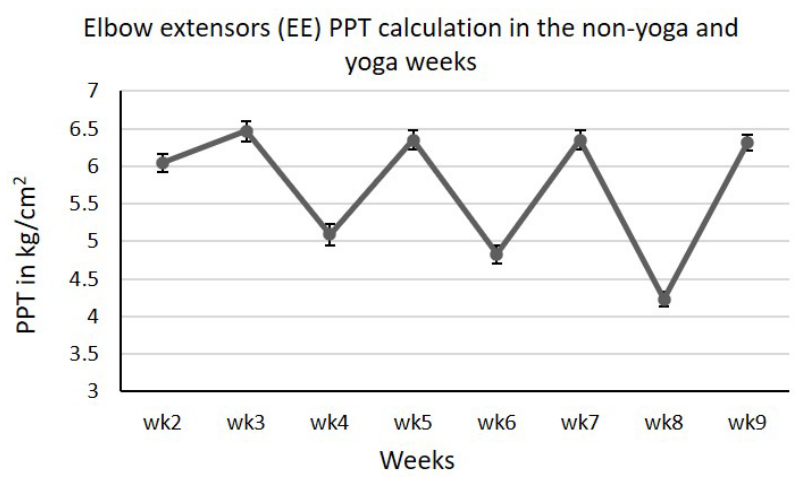

Figure 5. Elbow extensors (EE) PPT calculation in the non-yoga and yoga weeks

Yoga after the PROM and FROM MVICs (weeks 3, 5, $7,9)$ were compared and showed statistically significant differences $(P \leq 0.05)$ between them for all four muscle groups are shown in Table 2.

The MVIC mean difference was increased with weeks. At the end of the 25\% MVIC ECC protocol (end of week 5), MVIC values were increased between 9.91 and $13.42 \%$ whereas, after the 35\% MVIC ECC protocol (end of week 9), MVIC values were increased between 15.73 and $24.34 \%$. The overall percentage of increased MVIC value at the end of the study was between 30.53 and $43.12 \%$.

Table 2. MVIC comparison between yoga after PROM and yoga after FROM exercises using repeated measures one-way ANOVA with Tukey's multiple comparison tests

\begin{tabular}{ccccc}
\hline weeks & EF & EE & KF & KE \\
\hline & MD (sig.) & MD (sig.) & MD (sig.) & MD (sig.) \\
\cline { 2 - 5 } week1 vs week3 & $0.9133(\mathrm{~ns})$ & $1.96(* * *)$ & $1.967(* * * *)$ & $2.247(* * * *)$ \\
week3 vs week5 & $3.053(*)$ & $2.667(* * * *)$ & $2.447(* * * *)$ & $2.847(* * * *)$ \\
week5 vs week7 & $4.2(* * * *)$ & $3.98(* * *)$ & $2.847(* * * *)$ & $3.747(* * * *)$ \\
week7 vs week9 & $4.967(* * *)$ & $4.553(* * *)$ & $4.181(* * * *)$ & $4.581(* * * *)$ \\
week3 vs week7 & $7.253(* * * *)$ & $6.647(* * * *)$ & $5.297(* * * *)$ & $6.593(* * * *)$ \\
week5 vs week9 & $9.163(* * * *)$ & $8.533(* * * *)$ & $7.027(* * * *)$ & $8.327(* * * *)$ \\
\hline
\end{tabular}

MVIC - Maximal Voluntary Isometric Contraction; PROM - partial range of motion; FROM - the full range of motion; EF - elbow flexors; EE -elbow extensors; KF - knee flexors; KE - knee extensors; week1 - before exercise protocol; week3 - yoga after PROM exercise with 25\% of MVIC; week5 - yoga after FROM exercise with 25\% of MVIC; week7 - yoga after PROM exercise with 35\% of MVIC; week9- yoga after FROM exercise with 35\% of MVIC; ns - not significance; * - significance; MD (sig.) - mean difference (significance) 


\section{Discussion}

Eccentric training is a more effective way to exercise muscles than concentric training as it develops greater muscle force (up to $20-60 \%$ greater than concentric), muscle size, strength, flexibility, low risk of injury, and better sports performance. But the eccentric contractions may induce muscle pain and soreness than concentric contractions [19-21].

The eccentric exercises generate greater hypertrophy and strength effects for both upper and lower body training. The eccentric exercises performed with higher loads induce neural adaptation while lower loading causes hypertrophy of the muscles. The use of one over the other depends on individual needs [21]. There are many ways to relieve exercise-induced muscle pain. One of them is to try rolling out on the mat and doing some yoga poses for sore muscles.

Yoga integrates deep breathing with slow, conscious stretching on the muscles after a workout. There are many different asanas in yoga that target all different parts of the body and focus on specific muscles. Performing certain yoga poses have many health benefits like increasing flexibility, mobility, reducing anxiety and depression, mood, stress, and, in many cases, all-natural pain relief [22]. In the present study, the participants were made to perform selected yoga poses only after the ECC protocol. For the question, should we do yoga before or after the ECC protocol, the answer depends on many factors. For example, if the exercise depends more on strength than flexibility, then we would rather do it after the exercise. The general belief is that yoga can be best performed after a strengthening exercise where we can focus all the muscle groups used and stretched. This may help to recover faster and can also aid in bringing the heart rate back down and relax.

The study results showed that yoga performed after the PROM ECC protocol on the elbow and knee showed less pain, DOMS, and muscle strength when compared to the FROM ECC protocol without yoga poses. The ECC protocols performed without yoga poses showed more pain and DOMS. The participants performed mild (25\% of MVIC) to moderate (35\% of MVIC) intensity ECC protocols and the yoga poses after ECC protocol was also effective in relieving the muscle pain and DOMS. This could be due to the amount of intensity and repetition used in the PROM and FORM ECC exercise protocol in this study.

The evidence suggests that the intensity used during the ECC exercise may also affect the muscle damage response [22-25]. The participants that performed the ECC protocol with a smaller load (25\% of MVIC) felt less pain and DOMS than the ECC protocol with greater load (35\% of MVIC). Greater load and ROM ECC induce pain and DOMS whereas smaller load and ROM ECC showed less pain and DOMS.
In the FROM ECC protocol with greater load, the muscles were stretched and it led to muscle damage, pain, and DOMS. In the PROM ECC protocol with lesser load, the muscles were not stretched and caused minimal or no muscle damage, less pain, and DOMS. In this study, many factors like ROM, intensity, number of contractions, type of muscle contractions (ECC or CON), muscle groups exercised (flexors or extensors), and with or without yoga have influenced to determine the level of pain and DOMS. The participants who performed yoga poses after ECC protocol perceived mild or no pain on elbow flexors and knee extensors. The participants who performed ECC protocol without yoga poses perceived moderate pain on elbow extensors and knee flexors.

With a group of untrained subjects, Wirth et al. [21] studied the effects of eccentric training on the lower body maximal strength and speed-strength (power). The training group was made to do three lower body strength sessions using a 45-degree unilateral leg press for each week. This was carried out for six weeks. This training group was compared with another control group that did not do the training. It was observed, at the end of six weeks, the eccentric strength improved by $28.2 \%$ and the absolute strength by $31.1 \%$ for the training group. In the current study, the muscle strengths (MVIC) were increased in all the muscle groups studied. The FROM ECC protocol with $35 \%$ of MVIC increased the muscle strength compared to the PROM ECC protocol with 25\% of MVIC. In general, yoga poses involve many muscle groups of the body. Certain yoga poses work on specific muscle groups along with adjacent muscles. Child, thread the needle, eagle arms, cow face pose, and hands overhead in wide-legged forward bend poses were performed in the study for 20-30 mins. The selected yoga poses involved all the muscle groups studied (elbow and knee) in this study. One of the challenges was to distinguish the effect of yoga on elbow and knee muscle groups alone. Studied muscle groups were limited to elbow and knee joints. Participants did not perform yoga poses before the ECC protocol. These were the limitation of the study.

\section{Conclusions}

The performed yoga poses after ECC protocol reduced the pain and DOMS on the elbow extensors and knee flexors compared to elbow flexors and knee extensors. The Upper extremity (extensors) muscles were more prone to DOMS and pain compared to the lower extremity muscles. The performed yoga stretching poses after the eccentric exercise protocol reduced DOMS and pain in all the participants.

\section{Acknowledgments}

The author thanks the School of Biological Sciences 
(SBS), University of New England (UNE) for the start-up fund, and participants who allowed the publication of this study.

\section{Conflicts of Interest}

The author declares that there are no conflicts of interest regarding the publication of this paper.

\section{REFERENCES}

[1] K. Cheung, P. A. Hume, and L. Maxwell, "Delayed onset muscle soreness: treatment strategies and performance factors”, Sports Med, vol. 33, 2003, pp. 145-164. DOI: 10.2165/00007256-200333020-00005

[2] S. Murase, E. F. Terazawa, Queme, H. Ota, T. Matssuda, K. Hirate, Y. Kozaki, K. Katanosaka, T. Taguchi, H. Urai, and K. Mizumura, "Bradykinin and nerve growth factor play pivotal roles in muscular mechanical hyperalgesia after exercise (delayed-onset muscle soreness)", J Neurosci, vol. 30, 2010, pp. 3752-3761. DOI: 10.1523/JNEUROSCI.3803 $-09.2010$

[3] G. Howatson, and K. A. van Someren, "The prevention and treatment of exercise-induced muscle damage”, Sports Med, vol. 38, 2008, pp. 483-503. DOI: 10.2165/00007256-200838060-00004

[4] T. C. Chen, K. Nosaka, and P. Sacco, "Intensity of eccentric exercise, shift of optimum angle, and the magnitude of repeated-bout effect”, J Appl Physiol, vol. 102, 2007, pp. 992-999. DOI: 10.1152/japplphysiol.00425.2006

[5] D. A. Connolly, M. P. McHugh, O. I. Padilla-Zakour, L. Carlson, and S. P. Sayers, "Efficacy of a tart cherry juice blend in preventing the symptoms of muscle damage", $\mathrm{Br} \mathrm{J}$ Sports Med, vol. 40, 2006, pp. 679-683. DOI: 10.1136/bjsm.2005.025429

[6] R. Herbert, M. de Noronha, and S. Kamper, "Stretching to prevent or reduce muscle soreness after exercise", Cochrane Database Syst Rev, vol. 7, 2011, pp. CD004577. DOI: 10.1002/14651858.CD004577.pub3

[7] R. Torres, F. Ribeiro, J. Alberto Duarte, and J. M. H. Cabri, "Evidence of the physiotherapeutic interventions used currently after exercise-induced muscle damage: systematic review and meta-analysis”, Physical Therapy in Sport, vol. 13, 2012, pp. 101-114. DOI: 10.1016/j.ptsp.2011.07.005

[8] P. M. Barnes, B. Bloom, and R. L. Nahin, “Complementary and alternative medicine use among adults and children: United States”, Natl Health Stat Report, vol. 10, 2008, pp. 123. PMID: 15188733

[9] A. Lazaridou, A. Koulouris, J. K. Devine, M. Haack, R. N. Jamison, R. R. Edwards, and K. L. Schreiber, "Impact of daily yoga-based exercise on pain, catastrophizing, and sleep amongst individuals with fibromyalgia”, Journal of pain research, vol. 12, 2019, pp. 2915-2923. DOI: 10.2147/JPR.S210653
[10] N. Pasyar, N. Barshan Tashnizi, P. Mansouri, and S. Tahmasebi, "Effect of yoga exercise on the quality of life and upper extremity volume among women with breast cancer related lymphedema: A pilot study”, Eur J Oncol Nurs, vol. 42, 2019, pp. 103-109. DOI: 10.1016/j.ejon.2019.08.008

[11] A. A. Schmid, C. A. Fruhauf, J. L. Sharp, M. Van Puymbroeck, M. J. Bair, and J. D. Portz, "Yoga for People with Chronic Pain in a Community-Based Setting: A Feasibility and Pilot RCT", Journal of evidence-based integrative medicine, vol. 24, 2019, $2515690 X 19863763$. https://doi.org/10.1177/2515690X19863763

[12] D. G. Chang, J. A. Holt, M. Sklar, and E.J. Groessl, "Yoga as a treatment for chronic low back pain: A systematic review of the literature", Journal of orthopedics and rheumatology, vol. 3, 2016, pp. 1-8. PMID: 27231715

[13] M. J. Polsgrove, B. M. Eggleston, and R. J. Lockyer, "Impact of 10-weeks of yoga practice on flexibility and balance of college athletes", International journal of yoga, vol. 9, 2016, pp. 27-34. doi: 10.4103/0973-6131.171710

[14] N. P. Gothe, and E. McAuley, "Yoga Is as Good as Stretching-Strengthening Exercises in Improving Functional Fitness Outcomes: Results From a Randomized Controlled Trial”, J Gerontol A Biol Sci Med Sci, vol. 71, 2016, pp. 406-411. DOI: 10.1093/gerona/glv127

[15] S. K. Gupta, and S. Aparna, "Effect of Yoga Ocular Exercises on Eye Fatigue”, Int J Yoga, vol. 13, 2020, pp. 76-79. doi: 10.4103/ijoy.IJOY_26_19

[16] M. S. Ha, Y. H. Baek, J. W. Kim, and D. Y. Kim, “Effects of yoga exercise on maximum oxygen uptake, cortisol level, and creatine kinase myocardial bond activity in female patients with skeletal muscle pain syndrome”, Journal of physical therapy science, vol. 27, 2015, pp. 1451-1453. doi: 10.1589/jpts.27.1451

[17] R. B. Budhi, S. Payghan, and S. Deepeshwar, "Changes in Lung Function Measures Following Bhastrika Pranayama (Bellows Breath) and Running in Healthy Individuals", International journal of yoga, vol. 12, 2019, pp. 233-239. doi: 10.4103/ijoy.IJOY_43_18

[18] C. A. Boyle, S. P. Sayers, B. E. Jensen, S. Headley, and T. M. Manos, "The effects of yoga training and a single bout of yoga on delayed onset muscle soreness in the lower extremity”, Journal of Strength and Conditioning Research, vol. 18, 2004, pp. 723-729. DOI: 10.1519/14723.1

[19] C. J. Cook, C. B. Martyn, and P. K. Liam, "Three Weeks of Eccentric Training Combined with Overspeed Exercises Enhanced Power and Running Speed Performance Gains in Trained Athletes”, Journal of Strength and Conditioning Research, vol. 27, 2013, pp. 1280-1286. DOI: 10.1519/JSC.0b013e3182679278

[20] J. Mike, M. K. Chad, and K. Len, "How to Incorporate Eccentric Training into a Resistance Training Program”, Strength and Conditioning Journal, vol. 37, 2015, pp. 5-17. DOI: $10.1519 /$ SSC.0000000000000114

[21] K. Wirth, M. Keiner, E. Szilvas, H. Hartmann, and A. Sander, "Effects of Eccentric Strength Training on Different Maximal Strength and Speed-Strength Parameters of the Lower Extremity", Journal of strength and conditioning research, vol. 29, 2015, pp. 1837-1845. DOI: $10.1519 /$ JSC. 0000000000000528 
[22] D. Chapman, M. Newton, M. R. McGuigan, and K. Nosaka, "Effect of lengthening contraction velocity on muscle damage of the elbow flexors”, Med Sci Sports Exerc, vol. 40, 2008, pp. 926-933. DOI: 10.1249/MSS.0b013e318168c82d

[23] A. G. Fochi, F. Damas, R. Berton, I. Alvarez, M. Miquelini, T. F. Salvini, and C. A. Libardi, "Greater eccentric exercise-induced muscle damage by large versus small range of motion with the same end-point”, Biology of sport, vol. 33, 2016, pp. 285-289. doi: 10.5604/20831862.1208480

[24] K. Nosaka, M. Newton, P. Sacco, D. Chapman, and A.
Lavender, "Partial protection against muscle damage by eccentric actions at short muscle lengths", Medicine and science in sports and exercise, vol. 37, 2005, pp. 746-753. DOI: 10.1249/01.mss.0000162691.66162.00

[25] Gordon. E. Barkwell, James. P. Dickey, "The Effects of Plyometric Warm-up on Lower Limb Muscle Activity and Time to $10 \mathrm{~m}$ in the Backstroke Swimming Start”, International Journal of Human Movement and Sports Sciences, vol. 6, 2018, pp. 55 - 62. DOI: 10.13189/saj.2018.060302 